\title{
Polylactic acid as a suitable material for 3D printing of protective masks in times of COVID-19 pandemic
}

\author{
Eva Vaňková ${ }^{\text {Corresp., } 1}$, Petra Kašparová ${ }^{1}$, Josef Khun ${ }^{1}$, Anna Machková ${ }^{1}$, Jaroslav Julák ${ }^{1,2}$, Michal Sláma ${ }^{3}$, Jan Hodek \\ ${ }^{4}$, Lucie Ulrychová ${ }^{4}$, Jan Weber ${ }^{4}$, Klára Obrová ${ }^{5}$, Karin Kosulin ${ }^{5}$, Thomas Lion $^{5,6}$, Vladimír Scholtz $^{1}$ \\ 1 Department of Physics and Measurements, University of Chemistry and Technology Prague, Prague, Czech Republic \\ 2 Institute of Immunology and Microbiology, First Faculty of Medicine, Charles University and General University Hospital, Prague, Czech Republic \\ 3 Faculty of Science, University of Hradec Kralove, Hradec Králové, Czech Republic \\ 4 Institute of Organic Chemistry and Biochemistry of the Czech Academy of Science, Prague, Czech Republic \\ ${ }^{5}$ Children's Cancer Research Institute, Vienna, Austria \\ 6 Department of Pediatrics, Medical University of Vienna, Vienna, Austria
}

Corresponding Author: Eva Vaňková

Email address: Eva.Vankova@vscht.cz

A critical lack of personal protective equipment has occurred during the COVID-19 pandemic. Polylactic acid (PLA), a polyester made from renewable natural resources, can be exploited for 3D printing of protective face masks using the Fused Deposition Modelling technique. Since the possible high porosity of this material raised questions regarding its suitability for protection against viruses, we have investigated its microstructure using scanning electron microscopy and aerosol generator and photometer certified as the test system according to the standards EN 143 and EN 149. Moreover, the efficiency of decontaminating PLA surfaces by conventional chemical disinfectants including $96 \%$ ethanol, $70 \%$ isopropanol, and a commercial disinfectant containing $0.85 \%$ sodium hypochlorite has been determined. We confirmed that the structure of PLA protective masks is compact and can be considered a sufficient barrier protection against particles of a size corresponding to microorganisms including viruses. Complete decontamination of PLA surfaces from externally applied Staphylococcus epidermidis, Escherichia coli, Candida albicans and SARS-CoV-2 was achieved using all disinfectants tested, and human adenovirus was completely inactivated by sodium hypochlorite-containing disinfectant. Natural contamination of PLA masks worn by test persons was decontaminated easily and efficiently by ethanol. No disinfectant caused major changes to the PLA surface properties, and the pore size did not change despite severe mechanical damage of the surface. Therefore, PLA may be regarded as a suitable material for 3D printing of protective masks during the current or future pandemic crises. 
1 Polylactic acid as a suitable material for 3D printing of

2 protective masks in times of COVID-19 pandemic

3 Eva Vaňková ${ }^{1}$, Petra Kašparová ${ }^{1}$, Josef Khun ${ }^{1}$, Anna Machková ${ }^{1}$, Jaroslav Julák ${ }^{1,2}$, Michal

4 Sláma $^{3}$, Jan Hodek ${ }^{4}$, Lucie Ulrychová ${ }^{4}$, Jan Weber ${ }^{4}$, Klára Obrová ${ }^{5}$, Karin Kosulin ${ }^{5}$, Thomas

5 Lion $^{5,6}$, Vladimír Scholtz ${ }^{1}$

$6 \quad{ }^{1}$ Department of Physics and Measurements, University of Chemistry and Technology, Prague,

7 Czech Republic

$8{ }^{2}$ Institute of Immunology and Microbiology, First Faculty of Medicine, Charles University and

9 General University Hospital, Prague, Czech Republic

$10 \quad{ }^{3}$ Faculty of Science, University of Hradec Kralove, Hradec Králové, Czech Republic

$11{ }^{4}$ Institute of Organic Chemistry and Biochemistry of the Czech Academy of Science, Prague,

12 Czech Republic

$13{ }^{5}$ Children's Cancer Research Institute, Vienna, Austria

$14{ }^{6}$ Department of Pediatrics, Medical University of Vienna, Vienna, Austria

16 Corresponding Author:

17 Eva Vaňková ${ }^{1}$

18 Technická 5, Prague 6 - Dejvice, 166 28, Czech Republic

19 E-mail address: Eva.Vankova@vscht.cz

\section{Abstract}

A critical lack of personal protective equipment has occurred during the COVID-19 pandemic. Polylactic acid (PLA), a polyester made from renewable natural resources, can be exploited for 3D printing of protective face masks using the Fused Deposition Modelling technique. Since the possible high porosity of this material raised questions regarding its suitability for protection against viruses, we have investigated its microstructure using scanning electron microscopy and aerosol generator and photometer certified as the test system according to the standards EN 143 and EN 149. Moreover, the efficiency of decontaminating PLA surfaces by conventional chemical disinfectants including $96 \%$ ethanol, $70 \%$ isopropanol, and a commercial disinfectant containing $0.85 \%$ sodium hypochlorite has been determined. We confirmed that the structure of PLA protective masks is compact and can be considered a sufficient barrier protection against particles of a size corresponding to microorganisms including viruses. Complete decontamination of PLA surfaces from externally applied Staphylococcus epidermidis, Escherichia coli, Candida albicans and SARS-CoV-2 was achieved using all disinfectants tested, and human adenovirus was completely inactivated by sodium hypochlorite-containing disinfectant. Natural contamination of PLA masks worn by test persons was decontaminated easily and efficiently by ethanol. No disinfectant caused major changes to the PLA surface properties, and the pore size did not change despite severe mechanical damage of the surface. 
39 Therefore, PLA may be regarded as a suitable material for 3D printing of protective masks

40 during the current or future pandemic crises.

\section{Introduction}

42 COVID-19 (coronavirus disease 2019) is the designation of the disease caused by the SARS$\mathrm{CoV}-2$ infection. The World Health Organization (WHO) declared this epidemic a global pandemic affecting the whole world on March 11, 2020.

The infection by SARS-CoV-2 was confirmed for the first time in Wuhan, China, but had a huge impact also in Europe and later in North and South America. Lombardy, Italy was the most severely affected region in Europe. Due to the risk of health care system collapse, the Italian government ordered a nationwide lockdown (Spinelli and Pellino 2020). Several studies showed that SARS-CoV-2, similarly to SARS-CoV-1, remains infectious for hours and days in aerosols and on surfaces, respectively (Chin et al. 2020; Kampf et al. 2020; van Doremalen et al. 2020), emphasizing the need for efficient virucidal disinfection. The number of patients suffering from COVID-19 disease and the enormous rate of infection spread caused serious complications in many countries, including a desperate lack of protective equipment (Swennen et al. 2020). Sufficient production and distribution of protective equipment has been crucial for sustaining patient care during the pandemic. The current unsatisfactory situation regarding protective equipment in the USA has been described by Ranney et al. (2020). Because of the lack of protective equipment including face masks, extended manufacturing facilities have become very important for supporting the health care system. In this regard, the production of protective masks using 3D printing has proven very promising. This technology, often based on Fused Deposition Modelling (FDM) due to its cost and technical benefits, has found various applications in the manufacturing of medical devices such as prosthetic and dental implants or scaffolds in tissue engineering (Roopavath and Kalaskar 2017; Tack et al. 2016). The properties of 3D-printed objects render this technology attractive for manufacturing of protective masks. FDM provides adequate dimensional control, good surface finish and adaptability to use a variety of thermoplastic polymer filaments. The technology is based on high-temperature sintering of filaments and subsequent solidification of the printed product at room temperature. The polymers most commonly used for FDM are acrylonitrile-butadiene-styrene copolymers, polycarbonate, polyethylene terephthalate glycol (PETG) and polylactic acid (PLA) (Chadha et al. 2019; Ngo et al. 2018). Due to its unique properties, PLA is one of the most attractive materials for $3 \mathrm{D}$ printing. Its main advantages include low printing temperatures of $200-210^{\circ} \mathrm{C}$, smooth appearance, low toxicity and favorable mechanical properties, especially a low warping effect and high geometric resolution (Pajarito et al. 2019; Vicente et al. 2019). PLA is a biodegradable linear aliphatic polyester produced from renewable natural resources such as corn, wheat or sweet sorghum (Nampoothiri et al. 2010). Nagarajan et al. (2016) comprehensively reviewed its properties and applications. This polymer is produced by acidcatalyzed polycondensation of lactic acid monomers. Lactic acid of any chirality can be used, resulting in either poly-L-lactic acid, poly-D-lactic acid or poly-L,D-lactic acid (consisting of both isomers). Since L-lactic acid is the most common isomer in nature and is easily produced by 
79 lactic fermentation of various bio-wastes by bacteria (e.g. Lactobacillus spp.), it is also the most 80 commonly used precursor for PLA manufacturing. The possibility of biotechnological

81 production of the monomer significantly decreases its price, making the production of PLA very 82 cheap. The glass transition temperature of PLA ranges between 50 and $80{ }^{\circ} \mathrm{C}$, and the melting 83 temperature reaches approximately $175^{\circ} \mathrm{C}$. Due to its natural precursor, PLA is easily 84 biodegradable, e.g. by thermal decomposition, enzymatic digestion, oxidation or photolysis. 85 Ghorpade et al. (2001) studied the outcome of PLA-composting for 90 days and found that the 86 compound was degraded by $70 \%$. The use of PLA is limited by its poor thermal stability and 87 easy hydrolysis - it degrades more easily than other aliphatic polyesters. Nevertheless, PLA has 88 found many applications in diverse areas including the packaging industry as a food packaging 89 polymer for short shelf life products, the pharmaceutical industry for controlled drug delivery formulations and for tissue regeneration, and agriculture for better herbicide delivery management without negative effects on crop yield (Auras et al. 2004; Aziz et al. 2020; FartoVaamonde et al. 2019).

Protective masks made by 3D printing from PLA are designed for repeated use, requiring frequent cleaning and disinfection. The low glass transition temperature and relatively low melting point of PLA makes heat sterilization in an autoclave at $121^{\circ} \mathrm{C}$ impossible (McKeen 2014). The polymer can be sterilized using ethylene oxide, gamma radiation (Fleischer et al. 2020) or dry heat below $80^{\circ} \mathrm{C}$ for no more than $20 \mathrm{~min}$ (Zou et al. 2011). Fleischer et al. (2020) examined the changes of PLA properties after cleaning with chemical disinfectants such as Cidex Opa (Johnson \& Johnson) or chlorine solutions. Although these substances caused minor changes in stiffness and strength of 3D-printed PLA, 3D printing at appropriate conditions makes PLA objects mechanically amenable to cleaning and reuse. However, surface porosity of 3Dprinted PLA medical tools should be minimized to prevent exposure of users to residual disinfectants by inhalation or skin contact. Oth et al. (2019) studied PLA object sterilization by low-temperature hydrogen peroxide gas plasma in the commercially available Sterrad ${ }^{\circledR}$ apparatus (Johnson \& Johnson). They observed only sub-millimeter deformations induced by this process, rendering it suitable for sterilization in different areas including surgical applications. In contrast to conventional steam autoclaving, sterilization by hydrogen peroxide prevents deformation of 3D-printed objects made from PLA or PETG. Swennen et al. (2020) presented a prototype of reusable custom-made 3D-printed face masks (produced by a selective laser sintering technique) from polyamide composite components. The authors proposed

112 EXCEL, containing didecyldimethylammonium chloride and chlorhexidine digluconate.

113 Nevertheless, material leakage and virus decontamination of the reusable face mask components

114 have not been tested upon one or more disinfection cycles.

115 In the present study, we have investigated FDM 3D-printed PLA structure and porosity after 116 exposure to common chemical disinfectants including ethanol, isopropanol and a commercial 117 disinfectant containing sodium hypochlorite, which are easily accessible. In addition, we examined the efficiency of PLA disinfection after artificial contamination with bacteria 
119 (Staphylococcus epidermidis, Escherichia coli), a yeast fungus (Candida albicans), viruses

120 (SARS-CoV-2 and human adenovirus - HAdV) or natural contamination by wearing the masks.

\section{Materials \& Methods}

\section{PLA material and masks preparation by 3D printing}

123 Polylactic acid (PLA) was purchased in the form of filament for FDM 3D printing from Shenzen 124 Creality 3D Technology Co., LTD, China. Protective masks, circular plates (diameter of $10 \mathrm{~cm}$

125 and height of $0.2 \mathrm{~cm}$, printed vertically) and square carriers $(1 \times 1 \mathrm{~cm}, 0.2 \mathrm{~cm}$ high) (Fig. 1) were

126 prepared using a 3D printer (Prusa i3 MK3, Czech Republic). The printing template was

127 designed with Trimble Sketchup Pro, exported in a stereolithographic (stl) file (freely available

128 at the link https://www.facebook.com/groups/1346383268879783/files/) and used to print the

129 objects of investigation. The printing parameters were as follows: layer height $=0.3 \mathrm{~mm}$, shell

130 thickness (perimeter) $=0.4 \mathrm{~mm}$, bottom $/$ top thickness $=0.2 \mathrm{~mm}$, fill density $=10 \%$, print speed

$131=90 \mathrm{~mm} / \mathrm{s}$, extrusion temperature $=215^{\circ} \mathrm{C}$, platform temperature $=60^{\circ} \mathrm{C}$, filament flow $=95$

$132 \%$, machine nozzle size $0.4 \mathrm{~mm}$, the infill pattern was grid (i.e., linear tilted $45^{\circ}$ ) and the total

133 layers $=338$.

\section{Visualization of PLA mask structure using scanning electron microscopy}

135 The structure and porosity of PLA 3D-printed masks were examined using a scanning electron

136 microscope (SEM) Nova NanoSEM 450 (Fei, USA). Approximately $1 \times 1 \mathrm{~cm}$ pieces cut from

137 printed masks were completely air-dried and visualized by SEM. Since the material is very

138 sensitive to electron exposure, mild conditions had to be used, i.e. voltage of $5 \mathrm{kV}$ and low

139 vacuum. Images of each visualized position were captured by LVD detector at gradual

140 magnifications $2000 \times, 1000 \times, 500 \times, 100 \times$ (focusing on identical position), dwell time $5 \mu$ s and

141 spot size 4.5. The size of the pores between PLA filaments was measured and marked using a

142 SEM operating software (xT microscope Control v6.3.4 build 3233), provided by the SEM

143 manufacturer (Fei, USA). The SEM images shown in this study were selected as representative

144 visualizations of the PLA microstructure. The SEM analysis merely illustrates the 3D printed

145 PLA surface morphology, and gap width measurements are not analyzed statistically.

146 Visualization of PLA mask structure under stress conditions using scanning 147 electron microscopy

148 To investigate the impact of possible stress factors for PLA masks, cleaning with chemicals was 149 performed and exposure to wearing-associated contamination was simulated, as outlined below.

150 The effect of immersing in three chemical disinfectants [96\% ethanol, $70 \%$ isopropanol and the

151 commercial disinfectant and bleach SAVO Original, Unilever ČR s.r.o., Czech Republic

152 containing $0.85 \%$ sodium hypochlorite diluted with water (2:9)] was tested by repeated (5 x 15

$153 \mathrm{~min}$ ) cycles and long-term (24 hour) exposure.

154 The simulation of human impact on the PLA structure was performed as follows: extensive

155 exposure to fingers (to simulate incorrect application of the mask), abrasion with paper (minor 
156 mechanical stress) and dining fork (strong mechanical stress), immersion in $1.9 \%$ sodium

157 chloride solution for 4 hours (to simulate perspiration).

158 Short rinsing with $100 \%$ acetone was examined to investigate its effect on PLA surface

159 properties. After each treatment, completely dried PLA carriers were examined using SEM, as

160 described above.

\section{Aerosol particle passage through PLA material}

162 Surface contamination (and potential surface penetration) with infectious agents was simulated

163 by an aerosol generator and a photometer (Lorenz Meßgerätebau FMP 03) with a differential

164 pressure sensor (Supplementary Fig. 1), providing a suitable test system with stand for facial

165 masks and flat filter materials. The device was certified as a test system according to the

166 standards EN 143 (Respiratory protective devices - Particle filters - Requirements, testing,

167 marking), and EN 149 (Respiratory protective devices - Filtering half masks to protect against

168 particles - Requirements, testing, marking). A sample of the PLA material was attached in the

169 standardized testing cartridge and was sealed with silicone to prevent false positive detection of

170 penetrating particles passing along the edge of the PLA panel (Supplementary Fig. 2). The

171 cartridge was mounted into the Lorenz Meßgerätebau FMP 03 device, between the aerosol

172 generator and photometer. The aerosol generator produced a defined amount of aerosolized

173 paraffin oil, the test system passed it through the material, and the photometer situated on the

174 other side of the PLA sample measured the aerosol concentration, thereby indicating the

175 retention efficiency. An integrated differential pressure sensor was used to determine the

176 pressure loss during passage through the sample. The particle size distribution was

177 approximately $0.1-2 \mu \mathrm{m}$ (geometric mean $0.44 \mu \mathrm{m}$ ), which is close to the most frequently

178 observed penetrating particle size (Supplementary Fig. 3). The output of the aerosol generator

179 was set to $150 \%$ with flow $95 \mathrm{~L} / \mathrm{min}$, atomizer pressure 5 bar and oil temperature $60{ }^{\circ} \mathrm{C}$. The

180 test was performed for $270 \mathrm{~s}$.

181 Disinfection of PLA material artificially contaminated with bacteria and yeast 182 fungus

183 Wild strains of Staphylococcus epidermidis, Escherichia coli and Candida albicans were used as

184 representatives of gram-positive and gram-negative bacteria or yeast fungus, respectively. The

185 concentration of bacteria was adjusted to approximately $1 \times 10^{7}$ colony forming units (CFU) per

$186 \mathrm{~mL}$, the fungus concentration was $1 \times 10^{6} \mathrm{CFU} / \mathrm{mL}$. Each PLA carrier with a size of $1 \times 1 \mathrm{~cm}$ was

187 contaminated with $10 \mu \mathrm{L}$ of microbial suspension applied to the surface of carriers in $1 \mu \mathrm{L}$

188 droplets for $1 \mathrm{~h}$. The disinfection of contaminated carriers was carried out by immersing in $3 \mathrm{~mL}$

189 of $96 \%$ ethanol, $70 \%$ isopropanol, or $0.85 \%$ sodium hypochlorite (SAVO Original, Unilever ČR

190 s.r.o., Czech Republic) for 15 min. After evaporation of disinfectant solutions, the carriers were

191 immersed in $1 \mathrm{~mL}$ of sterile saline, vortexed, and the obtained suspensions were inoculated onto

192 appropriate agar plates. Blood agar was used for S. epidermidis, Müller-Hinton (Oxoid, Czech

193 Republic) agar for E. coli and Sabouraud agar (Oxoid, Czech Republic) for C. albicans. Samples

194 not exposed to treatment by disinfectants were used as controls. The inoculated plates were 
195 incubated at $37^{\circ} \mathrm{C}$ for $48 \mathrm{~h}$. Each experiment was done in triplicate, and results were obtained by

196 counting the average $\mathrm{CFU} / \mathrm{mL}$.

\section{Disinfection of PLA material artificially contaminated with viruses}

198

199

200

201

202

203

204

205

206

207

208

209

210

211

212

213

214

215

216

217

218

219

220

221

222

223

224

225

226

227

228

229

230

231

232

233

SARS-CoV-2, the causative agent of the COVID-19 pandemic, was isolated in a biosafety level 3 laboratory from a nasopharyngeal swab by inoculating Vero CCL81 cells (ECACC 84113001) and subsequent expansion by two additional passages in Vero CCL81 cells. Passage 3 was cleared by centrifugation at $1000 \mathrm{~g}$ for $5 \mathrm{~min}$, passed through a $0.45 \mu \mathrm{m}$ filter, and stored at -80 ${ }^{\circ} \mathrm{C}$ until use. In addition to SARS-CoV-2, inactivation of a stable DNA virus, the Human Adenovirus 2 ATCC VR-846 (HAdV) obtained from the American Type Culture Collection (ATCC) was assessed.

Similar to the previous set of experiments, PLA carriers of $1 \times 1 \mathrm{~cm}$ size were contaminated with $20 \mu \mathrm{L}$ of a SARS-CoV-2 suspension displaying a median tissue culture infectious dose (TCID50) of $10^{6} \mathrm{IU} / \mathrm{mL}$, which was applied to the surface of carriers in $1 \mu \mathrm{L}$ droplets. An additional set of carriers was covered with $50 \mu \mathrm{L}$ of HAdV suspension ( $10^{6}$ virus copies) spread evenly over the entire surface. The contaminated carriers were then immersed in $96 \%$ ethanol, $70 \%$ isopropanol or $0.85 \%$ sodium hypochlorite for $15 \mathrm{~min}$. Subsequently, residual viruses -if present- were washed from the dried surface using 180-200 $\mu \mathrm{L}$ PBS. The solution was used directly for infection of Vero-E6 cells (ATCC CRL-1586), in case of SARS-CoV-2, or A-549 human lung carcinoma cells (DSMZ ACC107 from German Collection of Microorganisms and Cell Cultures), in case of HAdV, respectively. Recovered SARS-CoV-2 was titrated by an immunofluorescence (IF) assay using a 1:2.5 serial dilution of Vero-E6 cells starting from 10 $\mu \mathrm{L}$. Vero-E6 cells were incubated for 72 hours at $37^{\circ} \mathrm{C}$ in a $\mathrm{CO}_{2}$ incubator prior to the IF assay. Briefly, medium was washed out, cells were fixed using $4 \%$ paraformaldehyde (PFA), cell membranes were perforated with $0.2 \%$ Triton-X100, and SARS-CoV-2 was labeled with primary mouse anti-SARS-CoV-2 antibody. Secondary anti-mouse antibody was conjugated with a $\mathrm{Cy} 3$ fluorophore and a fluorescent microscope (Olympus IX 81, Germany) was used for signal detection. In the case of HAdV, serial dilutions of virus inoculum were used to infect A-549 cells and the cytopathic effect (CPE) was determined using Motic AE21 Inverted Phase Contrast Microscope (Zeiss, Germany). The titers of both recovered viruses infection particles were determined as TCID50 and calculated using the Spearman-Kärber method (Kärber 1931; Spearman 1908). In addition, recovered HAdV genome copies were determined by real-time quantitative PCR (RQ PCR) as described previously (Lion et al. 2003) using the ABI Prism Fast 7500 Instrument (Thermo Fisher Scientific, MA, USA).

\section{Disinfection of PLA masks worn by test persons}

To investigate the feasibility of disinfecting PLA protective masks in practical use, three volunteers wore the protective masks of the same type for $4 \mathrm{~h}$. Thereafter, smears from one half of the inner (approximately $80 \mathrm{~cm}^{2}$ ) or outer surface (approximately $83 \mathrm{~cm}^{2}$ ) of each mask were performed using sterile cotton swabs. These samples served as a control for natural mask contamination by manual handling, direct skin contact and exhalation. Each cotton swab was

Peer) reviewing PDF | (2020:05:49258:2:0:NEW 24 Sep 2020) 
234 transferred into $1 \mathrm{~mL}$ of $0.9 \%$ saline in a microtube, vortexed and inoculated onto a blood agar

235 plate. Thereafter, the filters were removed from masks and the PLA skeletons of the masks were 236 immersed in $96 \%$ ethanol for $15 \mathrm{~min}$. After ethanol evaporation, cotton swab smears were taken 237 from the second halves of the inner and outer mask surfaces, inoculated onto agar plates, and 238 incubated at $37^{\circ} \mathrm{C}$ for $48 \mathrm{~h}$. The results were averaged and expressed as CFU $/ \mathrm{mL}$.

239 Results

240 Investigation of structure and porosity of 3D-printed PLA material

241 The structure and porosity of PLA masks produced by 3D printing were investigated by SEM.

242 Scanning electron micrographs of gaps between the PLA filaments were captured at four

243 different magnifications (Fig. 2). The PLA filament size determined was $312.8 \mu \mathrm{m}$ (

244 Supplementary Fig. 4) and its surface appeared macroscopically very smooth (Fig. 2A). Further

245 magnification showed only slight roughness of the surface and very small gaps between

246 filaments (Fig. 2B, 2C). Additional increase of magnification revealed connecting filaments of

247 PLA, resulting from the high temperature during 3D printing, with only very small pores $(6.049$

$248 \mu \mathrm{m}$ in size) in between. The pores appeared to be completely closed deeper in the carrier, as

249 observed at the highest magnification used (2000×) (Fig. 2D). To further test whether the pores

250 were indeed closed and prevented particles from passing through the printed mask, we

251 determined the number of paraffin oil aerosol particles displaying a size of $0.1-2 \mu \mathrm{m}$ using the

252 aerosol generator and photometer, certified as a test system according to the common standards.

253 Maximum pressure loss of the generated aerosol was detected, and absolutely no penetration

254 occurred even though the PLA sample was printed with a diameter of $10 \mathrm{~cm}$ (corresponds

255 approximately to the printed height of the masks) in the vertical position, simulated printing at a

256 lower temperature in the upper layers (on the z-axis).

257 Effect of ethanol, isopropanol and sodium hypochlorite on disinfection of PLA 258 material contaminated with bacteria, yeast fungus or viruses

259 The results of disinfection of artificially contaminated PLA are summarized in Tables 1 and 2.

260 Although the untreated PLA carriers were contaminated by highly concentrated bacterial

261 suspensions of $1 \times 10^{5} \mathrm{CFU} / \mathrm{mL}$, complete decontamination by all disinfectants used was

262 achieved. Single colonies were observed in the samples of $S$. epidermidis and E. coli disinfected

263 by isopropanol, but these isolated findings can reasonably be considered a contamination that

264 occurred after treatment of the samples. The disinfection of PLA carriers contaminated with $C$.

265 albicans $\left(4 \times 10^{4} \mathrm{CFU} / \mathrm{mL}\right)$ was complete in all cases.

266 Titers of SARS-CoV-2 and HAdV recovered from disinfected or untreated carriers were

267 determined by IF- and CPE-based assays, respectively. All disinfection agents tested showed

268 complete virucidal effects against SARS-CoV-2. Disinfectants per se exhibited a cytotoxic

269 effects on Vero-E6 cells (Supplementary Table 1), but this effect was eliminated by serial

270 dilutions during virus titer determination. HAdV infectivity was reduced by ethanol and

271 isopropanol, and completely abolished by sodium hypochlorite. Similar trends were observed by

272 RQ-PCR performed for detecting the HAdV genome copy numbers (Supplementary Table 2). 
273 Investigation of PLA structure after exposure to ethanol, isopropanol and sodium 274 hypochlorite

275 The effect of disinfectants on the PLA structure was investigated using SEM. PLA structure, 276 gaps between filaments, and the structure of pores after five 15 min cycles of immersing the carrier in different disinfectants are shown in Fig. 3. Treatment with ethanol (Fig. 3B) resulted in

278 slight melting of the PLA filaments, as compared with untreated PLA (Fig. 3A). The overall

279 PLA structure and surface did not change, but, interestingly, the gap size between the filaments

280 was reduced from the original $6 \mu \mathrm{m}$ to approximately $850 \mathrm{~nm}$ (Fig. 3B). This indicates that ethanol treatment may improve the PLA mask properties with regard to structure density. Similarly, isopropanol treatment did not significantly affect the PLA structure (Fig. 3C). Only slight melting was detectable, resulting in decreased gap size to $3.3-4 \mu \mathrm{m}$, in comparison to 6 $\mu \mathrm{m}$ in control samples. Moreover, the surface of filaments remained undamaged. Fig. 3D depicts the effect of sodium hypochlorite, which did not alter the surface of filaments, but precipitated disinfectant filled the gaps between them, while the gap size remained almost the same as in the control sample $(5-7 \mu \mathrm{m})$. Long-term treatment of PLA by immersion in disinfectants for 24 hours was also investigated using SEM (Fig. 4). The effect of long-term treatment with ethanol (Fig. 4B) was similar to repeated exposure to sodium hypochlorite (Fig. 3D), i.e. the gaps between filaments were significantly enlarged to $23.84 \mu \mathrm{m}$ (Fig. 4B), possibly filled with etched polymer. Investigation of aerosol particle passage through the PLA material after 24 hours in ethanol confirmed that the enlarged gaps were sealed, as no penetration was detected. PLA melting was also observed after prolonged isopropanol treatment (Fig. 4C). The gaps between filaments were sealed with the polymer in an irregular manner, resulting in variable gap sizes ranging from 1.3 to $4.1 \mu \mathrm{m}$. As in all previous tests with ethanol, the surface of PLA filaments remained unaffected. In contrast, long-term treatment with sodium hypochlorite damaged the surface of PLA filaments and revealed precipitation of the disinfectant on the surface (Fig. 4D). Similarly to short treatment with sodium hypochlorite, the gaps between filaments, ranging from 2 to $3.5 \mu \mathrm{m}$, were completely filled with precipitated sodium hypochlorite (Fig. 3D).

\section{Disinfection of PLA masks by ethanol upon wearing by test persons}

302

303

304

305

306

307

308

309

310

To complement the results of disinfection upon artificial contamination (Tables. 1 and 2), disinfection of PLA masks after natural use was investigated. The disinfection efficiency with ethanol $(96 \%)$ is summarized in Table 3. The microbial load detected on the inner surface of untreated masks varied significantly between different users, ranging from hundreds to thousands $\mathrm{CFU} / \mathrm{mL}$. Despite this variation, an average of $7 \mathrm{CFU} / \mathrm{mL}$ remained detectable after immersing the masks in ethanol for 15 minutes (short rinsing with ethanol was not sufficiently effective;Supplementary Fig. 5). On the outer surface of untreated masks, 50-150 CFU/mL were detected, and an average of $2 \mathrm{CFU} / \mathrm{mL}$ remained detectable after disinfection (Fig. 5).

\section{Visualization of PLA structure upon mechanical and chemical challenge}


311 The impact on the PLA material by finger contact, abrasion by paper or metal and treatment by 312 sodium chloride solution (mimicking perspiration) was analyzed using SEM (Fig. 6). Although

313 fingers may be greasy or sweaty, the contact did not cause any marks or alterations on the PLA

314 surface (Fig. 6A). Similarly, gentle mechanical abrasion with paper did not affect the material

315 (Fig. 6B). By contrast, intensive mechanical scraping with a dining fork significantly damaged

316 the PLA structure (Fig. 6C), leading to compression of PLA filaments, reduction of inter-

317 filament gaps, and shedding of PLA pieces (Fig. 6D). However, neither loosening of filaments,

318 nor increase in gap size or other deformations were observed. Soaking in sodium chloride

319 solution did not affect the structure, but salt crystals were present in the gaps between filaments

320 (Fig. 6E). In addition, the effect of acetone, which is known to damage PLA, was evaluated.

321 Virtually no gap was visible between filaments upon treatment, indicating that even short

322 exposure to acetone smoothens the structure and seals the pores (Fig. 6F).

\section{Discussion}

324 The unexpected and sudden spread of SARS-CoV-2 infection, which resulted in the COVID-19

325

326

327

328

329

330

331

332

333

334

335

336

337

338

339

340

341

342

343

344

345

346

347

348

349 pandemic, has led to a desperate shortage of personal protective equipment, especially among the frontline workers. Because of this problem, many people started helping each other by manufacturing facial protection equipment from commonly available resources. An intriguing possibility is the production of protective face masks using FDM, the most widespread technique of 3D printing. A variety of polymers are suitable for FDM, including biodegradable PLA as the most affordable and environmentally friendly material because of its natural origin (Ngo et al. 2018). Despite the potential benefits, the suitability of PLA-based materials for protection against viruses was questioned due to their possible high porosity. To the best of our knowledge, this report provides the first data addressing this issue by testing 3D-printed PLA masks (Fig. 1). The surface and other mechanical properties of products made from PLA or composite filaments were investigated previously (Graupner et al. 2009; Chi et al. 2018; Ivanov et al. 2019; Wang et al. 2016). However, the microstructure of 3D-printed PLA objects is highly dependent on the printing parameters, and it is not possible to predict the structure and porosity of a particular object based on published data. To investigate the surface properties of protective face masks made from PLA, examination of structure and porosity is required. We showed by SEM that 3Dprinted PLA masks have a compact structure, with small gaps between filaments. The gaps between individual filaments were $6 \mu \mathrm{m}$ wide, but higher magnification showed that the pores were not continuous within the PLA carrier (Fig. 2D) and were actually completely closed. This finding was supported by measurements of the filtering efficiency of PLA, which revealed completely blocked passage of nanometer-sized paraffin aerosol particles. The mask material can therefore be considered impermeable for particles displaying the size range tested, including the fungus, bacteria, and viruses investigated. In combination with the obligatory single-use filters complying with FFP2/3 standards, which are inserted into the mask, spreading of the smallest viruses can also be prevented. Moreover, short exposure to acetone resulted in smoothening of the PLA surface (Fig. 6F). 
350 A similar 3D-printed reusable face mask prototype was reported by Swennen et al. (2020). The

351

352

353

354

355

356

357

358

359

360

361

362

363

364

365

366

367

368

369

370

371

372

373

374

375

376

377

378

379

380

381

382

383

384

385

386

387

388

389

material (polyamide composite) and the printing method used (selective laser sintering technique) differ from the approach presented, but it provided a proof of principle for 3D printing of individualized 3D face masks with FFP2/3 filter membranes as a feasible and valuable alternative source for protective equipment. However, the authors of the cited study did not perform any virus decontamination testing of the reusable components of the face masks and were hence unable to assess the impact of repeated cycles of disinfection on the properties of the material. It was important therefore to determine the possibility of disinfecting the reusable face mask matrix.

While SARS-CoV-2, being an enveloped RNA virus, belongs to the less challenging pathogens in terms of disinfection, HAdV (non-enveloped DNA virus) is highly resistant to commonly used disinfectants (Gordon et al. 1993; Lion et al. 2020). Adenoviruses mostly cause infections with only mild symptoms in immunocompetent hosts (Lion 2019), but due to their exceptional stability provide a perfect model for testing the inactivation efficiency. In addition, we examined the disinfection of PLA material from contamination with bacteria (S. epidermidis and E. coli) and yeast fungus (C. albicans). These microorganisms are part of the human microbiome and their persistence on the protective mask surface poses a risk for infection and a health threat to mask users (Fisher and Shaffer 2014). All bacterial and fungal microorganisms studied were successfully disinfected using either $96 \%$ ethanol, $70 \%$ isopropanol or $0.85 \%$ sodium hypochlorite, after immersing contaminated PLA carriers in the respective disinfectant for 15 min (Table 1). Ethanol disinfected the PLA masks contaminated from using by humans (Fig. 5). In comparison to bacteria or fungi, viruses tend to be 1-2 orders of magnitude smaller, making them prone to enter deep into pores of the PLA material. Nevertheless, our data show that efficient disinfection of the PLA carriers from virus contamination is possible, as all tested disinfectants completely inactivated SARS-CoV-2 (Table 2). Treatment with sodium hypochlorite for 15 minutes also completely inactivated the highly resistant HAdV, while ethanol and propanol only led to reduced loads of infectious virus (Table 2). These data are in agreement with the reported sensitivity of both SARS-CoV-2 (Chin et al. 2020; Kampf et al. 2020) and HAdV to specific disinfectants (Gordon et al. 1993; Lion et al. 2020). The present findings therefore provide evidence that PLA material disinfection can be performed with comparable efficiency to other surfaces by appropriate exposure to individual disinfectants. The results obtained can conceivably also help design efficient disinfection protocols for protective face masks made from different materials.

Fleischer et al. (2020) examined the changes of PLA material after cleaning with chemical disinfectants (Cidex Opa, Johnson \& Johnson and chlorine solutions), revealing mild alterations in the stiffness and strength of 3D-printed PLA samples. However, the authors concluded that high-quality 3D-printed surfaces generated with appropriate printer settings permit cleaning and reuse of 3D-printed medical tools, without compromising their mechanical properties. The authors also stated that immersion in cleaning agents can lead to their absorption into the PLA structure. Thus, additional research is needed to establish efficient and safe chemical cleaning of

PeerJ reviewing PDF | (2020:05:49258:2:0:NEW 24 Sep 2020) 
390 various 3D-printed surfaces, to prevent health risks associated with tactile and inhalation

391 exposure to chemically cleaned materials.

392 In general, we observed that 5 cycles of PLA treatment for 15 minutes with alcohol-based

393 disinfectants resulted in decreased gap size between PLA filaments, without any remnants of

394 disinfectant visible by SEM. By contrast, sodium hypochlorite precipitate was retained in the

395 PLA structure, filling the gaps between PLA filaments. Disinfection of PLA masks with $0.85 \%$

396 sodium hypochlorite therefore requires further medical investigation to determine whether

397 exposure to the precipitate might be associated with any health risks. Long-term (24-hour)

398 treatment of PLA material with disinfectants resulted in partial melting of the filaments, but no

399 erosions of the material were observed (Fig. 4). Ethanol seems to be best suited for the

400 disinfection of PLA masks because it evaporates and does not require removal by rinsing.

401 Moreover, the barrier properties of the mask were not compromised even after long-term

402 exposure, as determined by aerosol challenge.

403 Although the surface of protective equipment should remain intact, inadvertent contacts with the

404 hands and fingers often occur, and the possibility of inappropriate handling has to be considered.

405 The pandemic setting requires medical staff to wear extensive protective equipment (e.g.

406 overalls, gloves, protective shields, and face masks). Such equipment, together with high

407 workload and stress, increases the body temperature and leads to excessive sweating. We

408 mimicked such conditions by mechanical and chemical treatment in order to evaluate alterations

409 of the protective masks. Touching the surface of the PLA material with fingers had no impact,

410 but intensive mechanical stress caused alteration of the PLA filament surface, without affecting

411 the inter-filament gap area. Treatment with sodium chloride (imitating perspiration and sweat)

412 showed salt crystallization in the gaps between filaments (Fig. 6E). Crystallized salt compounds,

413 such as sodium chloride or sodium hypochlorite (Figs. 3D and 4D), can cause discomfort by skin

414 irritation and itching. This issue was described in detail by Payne (2020) and Wollina (2020)

415 who stated that especially front-line workers obliged to wear a single face mask all day suffer

416 from these problems. The exploitation of PLA may solve this issue, because the fast and cheap

417 manufacturing of protective masks made from this material permits production on a large scale,

418 thereby facilitating more frequent mask changes. Additionally, 3D-printed protective PLA masks

419 are biodegradable, with relatively short decomposition time, thereby providing an

420 environmentally friendly solution.

\section{Conclusions}

422 This study shows that PLA material is suitable for protection against various microorganisms as

423 it is not permeable for submicroscopic particles. PLA can be efficiently disinfected from

424 bacteria, yeast fungus, and SARS-CoV-2 by commonly available chemical disinfectants such as

425 ethanol, isopropanol or sodium hypochlorite. However, contamination with HAdV, a highly

426 resistant representative of non-enveloped viruses, could only be completely removed with

427 sodium hypochlorite. PLA material is not altered by the immersion in disinfectant or by manual

428 handling. Possible skin irritation after the use of certain disinfectants needs to be carefully

429 evaluated. Single-use filters meeting the FFP2/3 standards are inserted into the mask structure 
430 and will be subject of further research and optimization. Overall, PLA can be recommended as 431 suitable material for the manufacturing of protective face masks at times of epidemic spread of 432 infections, such as the ongoing COVID-19 pandemic.

\section{Acknowledgements}

434 We thank the volunteers from the organization called "3D tiskem proti viru" (eng. version "3D printing against the virus"; https://www.3dtiskemprotiviru.cz/) as well as Pavel Kubíček, who printed 3D protective masks for people free of charge during the COVID-19 pandemic. We also thank Václav Čeřovský and Jiř́ Rybáček from IOCB of the CAS and AVEC CHEM s.r.o. for providing the laboratory environment and equipment enabling the project to be conducted.

\section{References} Biosci. 2004; 4(9): 835-864, https://doi.org/10.1002/mabi.200400043. polylactic acid (PLA). Polym. Test. 2020; 85: 106434-106464, https://doi.org/10.1016/j.polymertesting.2020.106434. manufacture loading of filaments and 3D printed PLA scaffolds with prednisolone and dexamethasone for tissue regeneration applications. Eur. J. Pharm. Biopharm. 2019; 141: 100110, https://doi.org/10.1016/j.ejpb.2019.05.018. Fisher EM, Shaffer RE. Considerations for recommending extended use and limited reuse of filtering facepiece respirators in health care settings. J. Occup. Environ. Hyg. 2014; 11: D115D128, https://doi.org/10.1080/15459624.2014.902954. 14(1): 011109-011118, https://doi.org/10.1115/1.4046120. sheets. Bioresour. Technol. 2001; 76(1): 57-61, https://doi.org/10.1016/S0960-8524(00)00077-8. Gordon YJ, Gordon RY, Romanowski E, Araullo-Cruz TP. Prolonged recovery of desiccated adenoviral serotypes 5, 8, and 19 from plastic and metal surfaces in vitro. Ophthalmology. 1993; 100(12): 1835-1840, https://doi.org/10.1016/S0161-6420(93)31389-8. (lactic acid)(PLA) composites: An overview about mechanical characteristics and application areas. Composites, Part A 2009; 40: 810-821, https://doi.org/10.1016/j.compositesa.2009.04.003. 
467 Chi H, Li W, Fan C, Zhang C, Li L, Qin Y, Yuan M. Effect of High Pressure Treatment on Poly 468 (lactic acid)/Nano-TiO2 Composite Films. Molecules 2018; 23: 2621-2632,

469 https://doi.org/10.3390/molecules23102621.

470 Chin A, Chu JTS, Perera MRA, Hui KPY, Yen H-L, Chan MCW, Peiris M, Poon LLM. Stability

471 of SARS-CoV-2 in different environmental conditions. Lancet Microbe MedRxiv 2020; 1(1):

472 E10, https://doi.org/10.1016/S2666-5247(20)30003-3.

473 Ivanov E, Kotsilkova R, Xia H, Chen Y, Donato RK, Donato K, Godoy AP, Di Maio R, Silvestre 474 C, Cimmino S, Angelov V. PLA/graphene/MWCNT composites with improved electrical and 475 thermal properties suitable for FDM 3D printing applications. Appl. Sci. 2019; 9: 1209-1223, 476 https://doi.org/10.3390/app9061209.

477 Kampf G, Todt D, Pfaender S, Steinmann E. Persistence of coronaviruses on inanimate surfaces 478 and its inactivation with biocidal agents. J. Hosp. Infect. 2020; 104(3): 246-251, 479 https://doi.org/10.1016/j.jhin.2020.01.022.

480 Kärber G. Beitrag zur kollektiven Behandlung pharmakologischer Reihenversuche. Archiv $f$. 481 experiment. Pathol. u. Pharmakol. 1931; 162: 480-483, https://doi.org/10.1007/BF01863914. 482 McKeen LW. Plastics used in medical devices. In: Handbook of polymer applications in 483 medicine and medical devices. William Andrew Publishing, 2014; 21-53,

484 https://doi.org/10.1016/B978-0-323-22805-3.00003-7.

485 Lion T. Adenovirus persistence, reactivation, and clinical management. FEBS Lett. 2019; 486 593(24):3571-3582. https://doi:10.1002/1873-3468.13576

487 Lion T, Baumgartinger R, Watzinger F, Matthes-Martin S, Suda M, Preuner S, Futterknecht B, 488 Lawitschka A, Peters C, Potschger U, Gadner H. Molecular monitoring of adenovirus in 489 peripheral blood after allogeneic bone marrow transplantation permits early diagnosis of 490 disseminated disease. Blood. 2003;102(3):1114-20, https://doi:10.1182/blood-2002-07-2152.

491 Lion T and Wold WSM. Adenovirus. In: DM Knipe, \& PM Howley (Eds.), Fields Virology, 7th 492 Edition. Philadelphia, PA, USA: Lippincott, Williams \& Wilkins, 2020 (in press).

493 Nagarajan V, Mohanty AK, Misra M. Perspective on polylactic acid (PLA) based sustainable 494 materials for durable applications: Focus on toughness and heat resistance. ACS Sustainable 495 Chem. Eng. 2016; 4(6): 2899-2916, https://doi.org/10.1021/acssuschemeng.6b00321.

496 Nampoothiri KM, Nair NR, John RP. An overview of the recent developments in polylactide 497 (PLA) research. Bioresour. Technol. 2010; 101(22): 8493-8501,

498 https://doi.org/10.1016/j.biortech.2010.05.092.

499 Ngo TD, Kashani A, Imbalzano G, Nguyen KT, Hui D. Additive manufacturing (3D printing): A 500 review of materials, methods, applications and challenges. Compos. Part B Eng. 2018; 143: 172501 196, https://doi.org/10.1016/j.compositesb.2018.02.012.

502 Oth O, Dauchot C, Orellana M, Glineur R. How to Sterilize 3D Printed Objects for Surgical 503 Use? An Evaluation of the Volumetric Deformation of 3D-Printed Genioplasty Guide in PLA 504 and PETG after Sterilization by Low-Temperature Hydrogen Peroxide Gas Plasma. Open. Dent. 505 J. 2019; 13: 410-417, 10.2174/1874210601913010410. 
506 Pajarito BB, Cayabyab CAL, Costales PAC, Francisco JR. Exfoliated graphite/acrylic composite 507 film as hydrophobic coating of 3D-printed polylactic acid surfaces. J. Coat. Technol. Res. 2019; 508 16: 1133-1140, https://doi.org/10.1007/s11998-019-00188-4.

509 Payne A. Covid-19: skin damage with prolonged wear of FFP3 masks. BMJ 2020; 369: m1743, 510 https://doi.org/10.1136/bmj.m1743.

511 Ranney ML, Griffeth V, Jha AK. Critical supply shortages - the need for ventilators and 512 personal protective equipment during the Covid-19 pandemic. N. Engl. J. Med. 2020; 382: e41, 513 https://doi.org/10.1056/NEJMp2006141.

514 Roopavath UK, Kalaskar DM. Introduction to 3D printing in medicine. In: 3D printing in 515 medicine. Woodhead Publishing, 2017; 1-20, https://doi.org/10.1016/B978-0-08-100717-

516 4.00001-6.

517 Spearman C. The method of 'right and wrong cases'('constant stimuli') without Gauss's

518 formulae. Br. J. Psychol. 1908; 2 (1904-1920): 227-242, https://doi.org/10.1111/j.2044-

519 8295.1908.tb00176.x.

520 Spinelli A, Pellino G. COVID-19 pandemic: perspectives on an unfolding crisis. Br. J. Surg. 521 2020; 107(7): 785-787, https://doi.org/10.1002/bjs.11627.

522 Swennen GRJ, Pottel L, Haers PE. Custom-made 3D-printed face masks in case of pandemic 523 crisis situations with a lack of commercially available FFP2/3 masks. Int. J. Oral. Maxillofac.

524 Surg. 2020; 49(5): 673-677, https://doi.org/10.1016/j.ijom.2020.03.015.

525 Tack P, Victor J, Gemmel P, Annemans L. 3D-printing techniques in a medical setting: a

526 systematic literature review. Biomed. Eng. Online. 2016; 15(1): 115-135,

527 https://doi.org/10.1186/s12938-016-0236-4.

528 Van Doremalen N, Bushmaker T, Moris DH, Phil M, Holbrook MG, Gamble A, Williamson BN, 529 Tamin A, Harcourt JL, Thornburg NJ, Gerber SI, Lloyd-Smith JO, de Wit E, Munster VJ.

530 Aerosol and surface stability of SARS-CoV-2 as compared with SARS-CoV-1. N. Engl. J. Med.

$5312020 ; 382:$ 1564-1567, https://doi.org/10.1056/NEJMc2004973.

532 Vicente C, Fernandes J, Deus A, Vaz M, Leite M, Reis L. Effect of protective coatings on the 533 water absorption and mechanical properties of 3D printed PLA. Fra. \& Int. Strut. 2019; 13: 748534 756, https://doi.org/10.3221/IGF-ESIS.48.68.

535 Wang M, Favi P, Cheng X, Golshan NH, Ziemer KS, Keidar M, Webster TJ. Cold atmospheric 536 plasma (CAP) surface nanomodified 3D printed polylactic acid (PLA) scaffolds for bone 537 regeneration. Acta Biomater. 2016; 46: 256-265, https://doi.org/10.1016/j.actbio.2016.09.030. 538 Wollina U. Challenges of COVID-19 pandemic for dermatology. Dermatol. Ther. 2020; e13430539 e13452, https://doi.org/10.1111/dth.13430.

540 Zou HT, Gan HL, Wang LX, Yi CH. Effect of wet-heat and dry-heat processing on mechanical 541 properties of PLA fibers. Adv. Mater. Res. 2011; 332-334: 870-873,

542 https://doi.org/10.4028/www.scientific.net/AMR.332-334.870. 


\section{Figure 1}

Objects made from PLA filaments using 3D printing by the FDM technology.

(A) PLA carriers $(1 \times 1 \mathrm{~cm})$. (B) Circular plate with a diameter of $10 \mathrm{~cm}$ (printed vertically). (C, D, E) Different types of PLA masks.

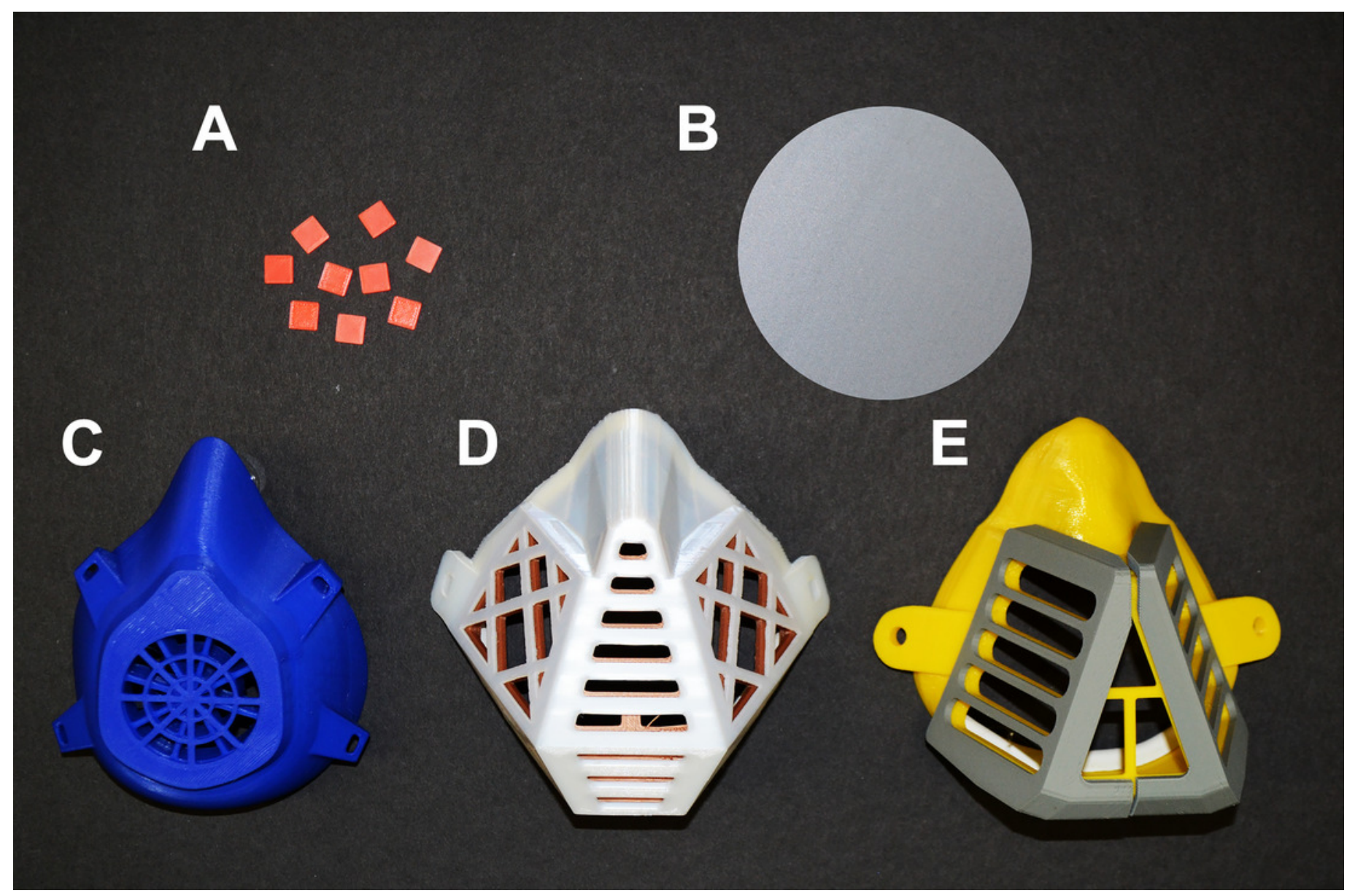




\section{Figure 2}

Scanning electron micrographs depicting the structure and porosity of PLA material used for 3D printing of protective masks.

(A) Magnification $100 x$, scale bar $500 \mu \mathrm{m}$. (B) Magnification 500x, scale bar $100 \mu \mathrm{m}$. (C) Magnification 1000x, scale bar $50 \mu \mathrm{m}$. (D) Magnification 2000x, scale bar $30 \mu \mathrm{m}$. The observed gaps were measured and marked by black lines. SEM parameters: low vacuum, 5 kV, LVD detector, dwell time $5 \mu \mathrm{s}$, spot size 4.5. Images were taken at various magnifications at the same position.

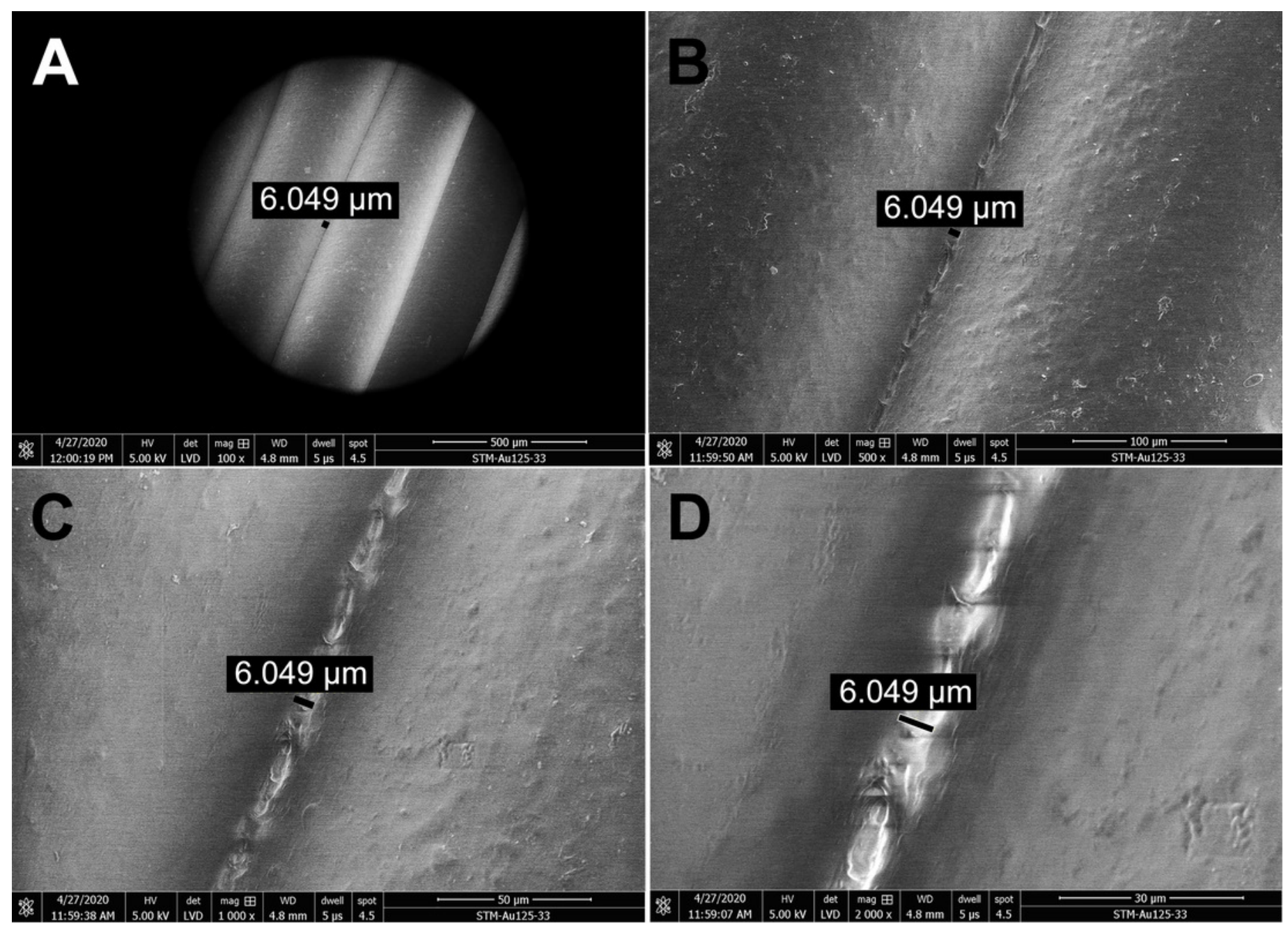




\section{Figure 3}

Scanning electron micrographs depicting the structure and porosity of PLA material after short repeated treatment ( 5 times $15 \mathrm{~min}$ ) with disinfectants.

(A) Untreated sample. (B) Sample treated with $96 \%$ ethanol. (C) Sample treated with $70 \%$ isopropanol. (D) Sample treated with $0.85 \%$ sodium hypochlorite. SEM parameters: low vacuum, $5 \mathrm{kV}$, LVD detector, dwell time $5 \mu \mathrm{s}$, spot size 4.5. Images were taken at $2000 \mathrm{x}$ magnification (scale bar $=30 \mu \mathrm{m}$ ) at the same position. The observed gaps were measured and marked by black lines.

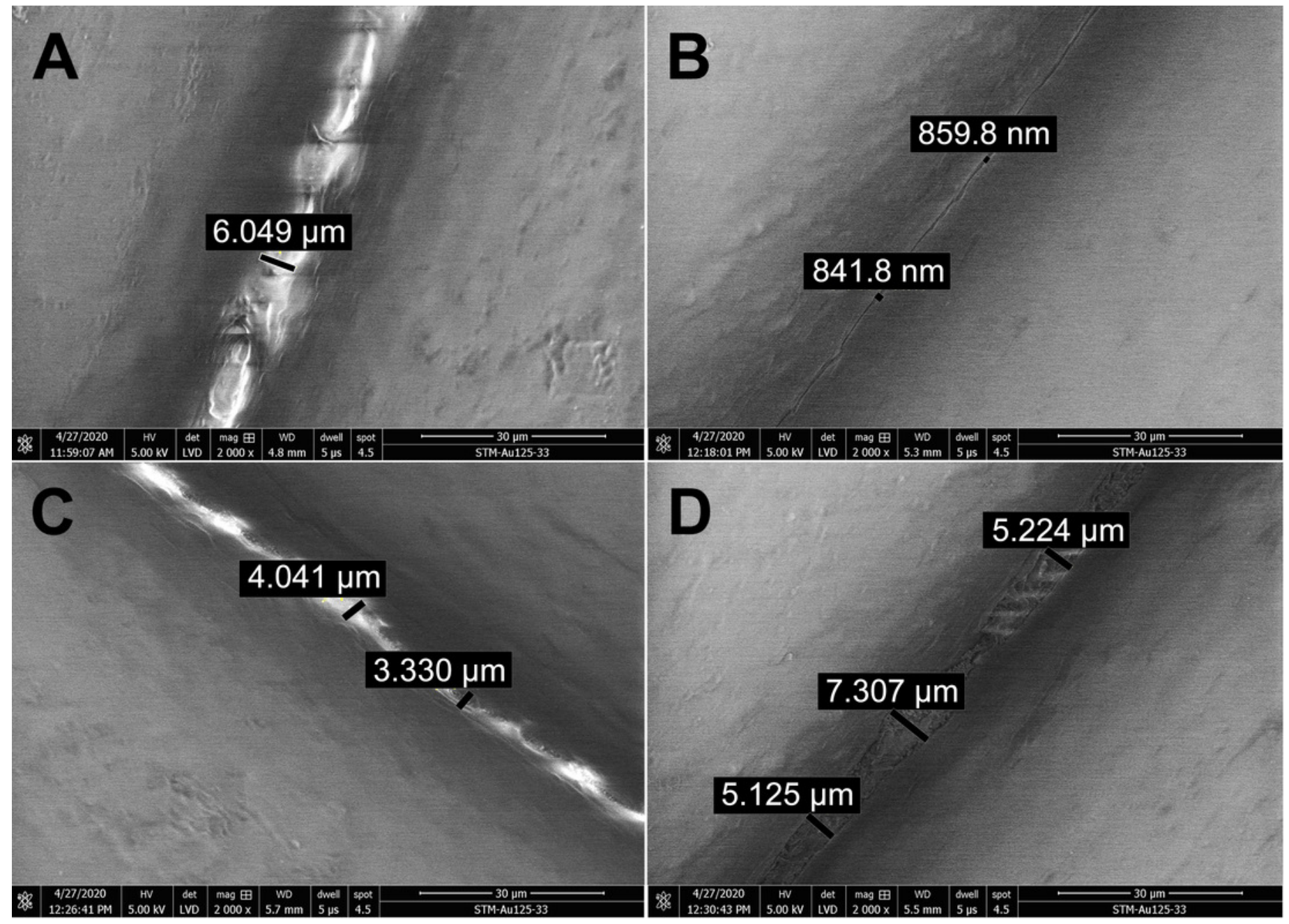




\section{Figure 4}

Scanning electron micrographs depicting the structure and porosity of PLA material after long-time treatment ( 24 hours) with disinfectants.

(A) Untreated sample. (B) Sample treated with 96\% ethanol. (C) Sample treated with 70\% isopropanol. (D) Sample treated with $0.85 \%$ sodium hypochlorite . SEM parameters: low vacuum, $5 \mathrm{kV}$, LVD detector, dwell time $5 \mu \mathrm{s}$, spot size 4.5. Images were taken at 2000x magnification (scale bar $=30 \mu \mathrm{m}$ ) at the same position. The observed gaps were measured and marked by black lines.

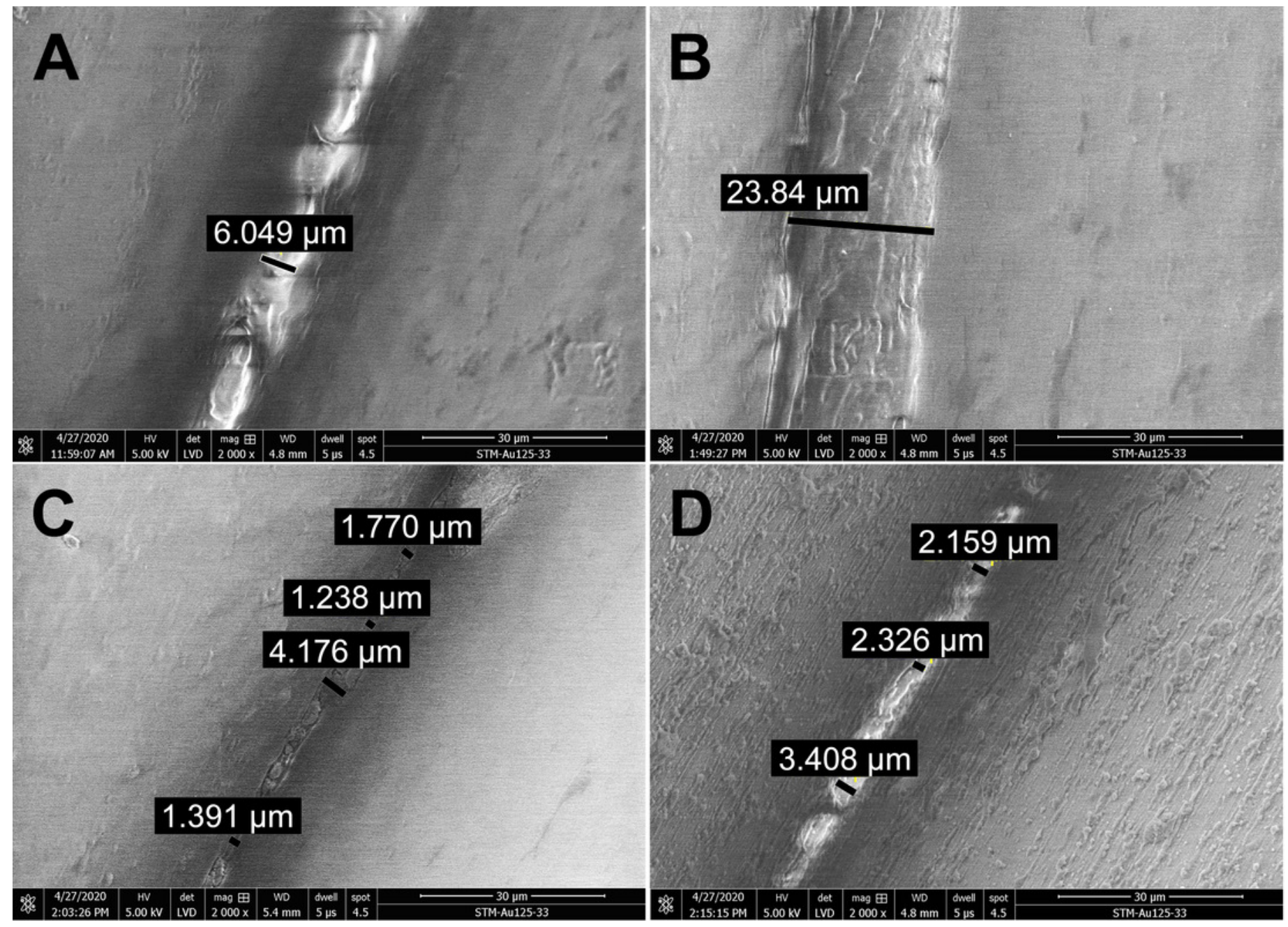




\section{Figure 5}

Effect of ethanol on disinfection of PLA masks contaminated by wearing for $4 \mathrm{~h}$.

A representative set of blood agar plates is displayed. The plates were inoculated with material collected from (A) inner surface of the mask before treatment; $(B)$ inner surface of the mask disinfected by immersion in ethanol for $15 \mathrm{~min}$; (C) outer surface of the mask before treatment; (D) outer surface of the mask disinfected by immersion in ethanol for 15 $\min$. 
A

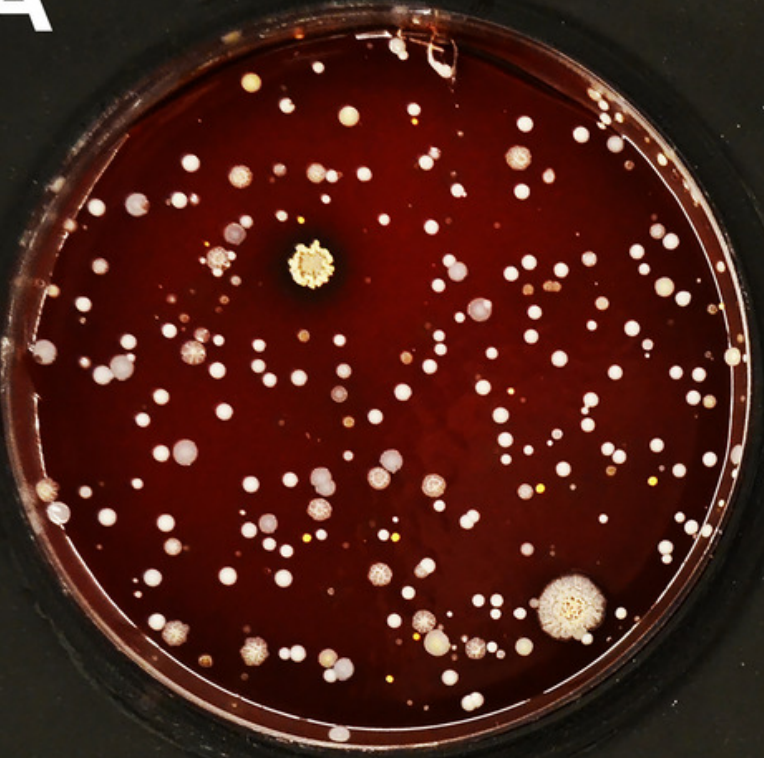

C

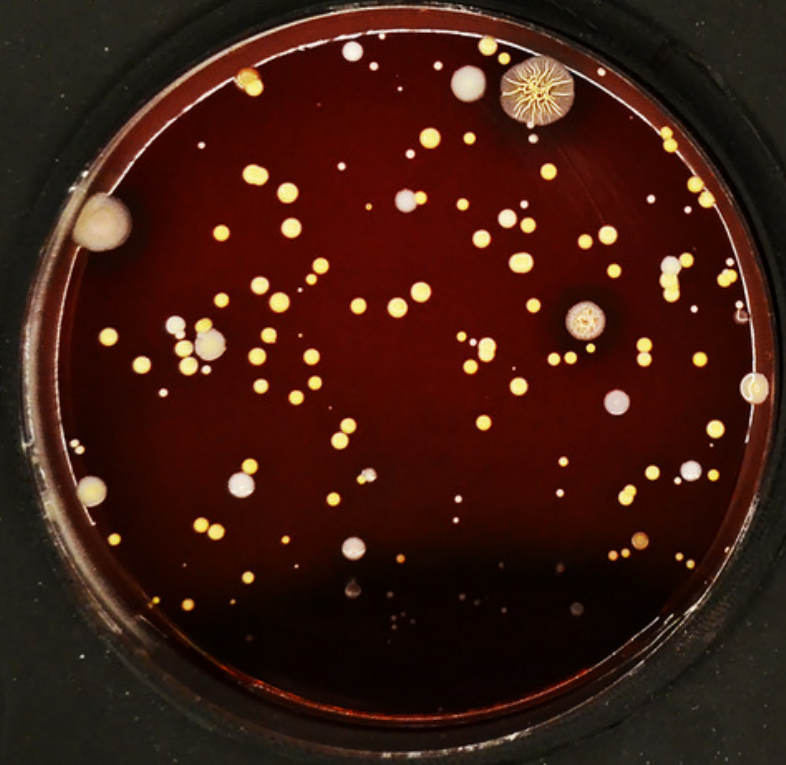

B

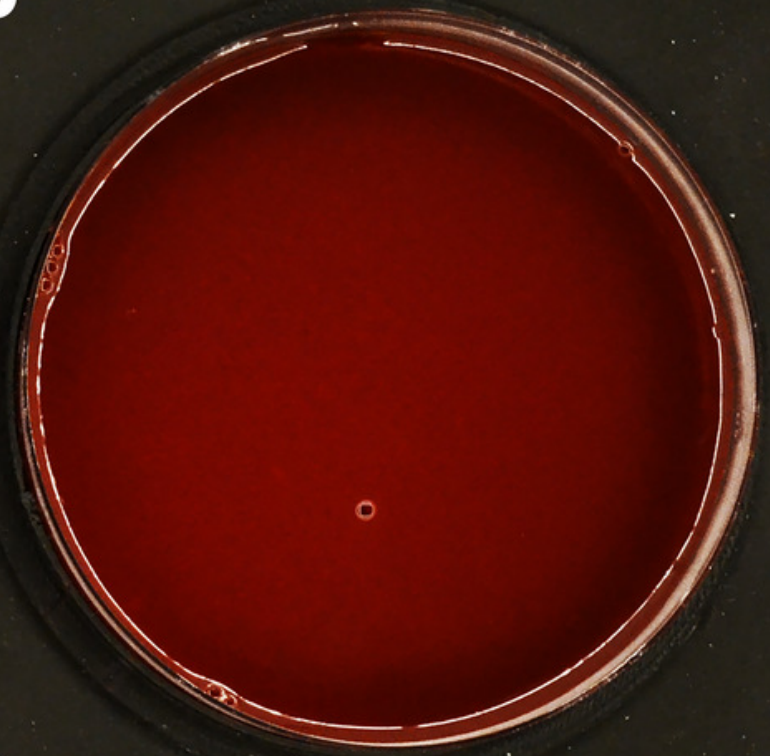

D

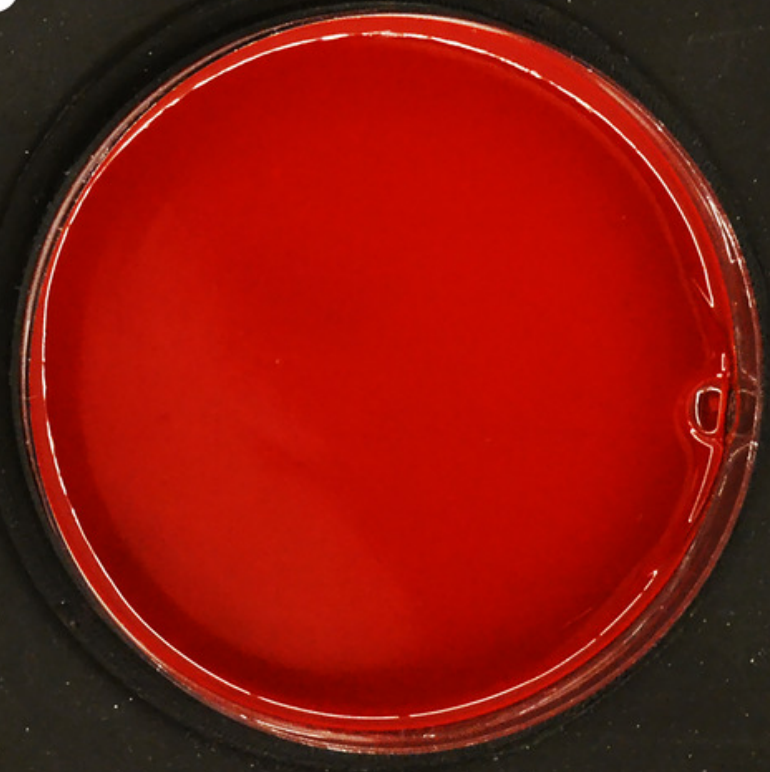




\section{Figure 6}

Scanning electron micrographs depicting the simulation of human impact on structure and porosity of PLA protective masks.

(A) Sample touched by finger. (B) Slightly mechanically stressed sample (paper abrasion). (C) Extremely mechanically stressed sample (scratching by dining fork). (D) Detail of a pore in extremely mechanically stressed sample (dining fork). (E) Sample after immersion in saline solution (perspiration and sweat simulation). (F) Sample after short rinsing with acetone. SEM parameters: low vacuum, $5 \mathrm{kV}$, LVD detector, magnification $100 \times$ or $500 x$, dwell time $5 \mu \mathrm{s}$, spot size 4.5, scale bar 500 or $100 \mu \mathrm{m}$. 


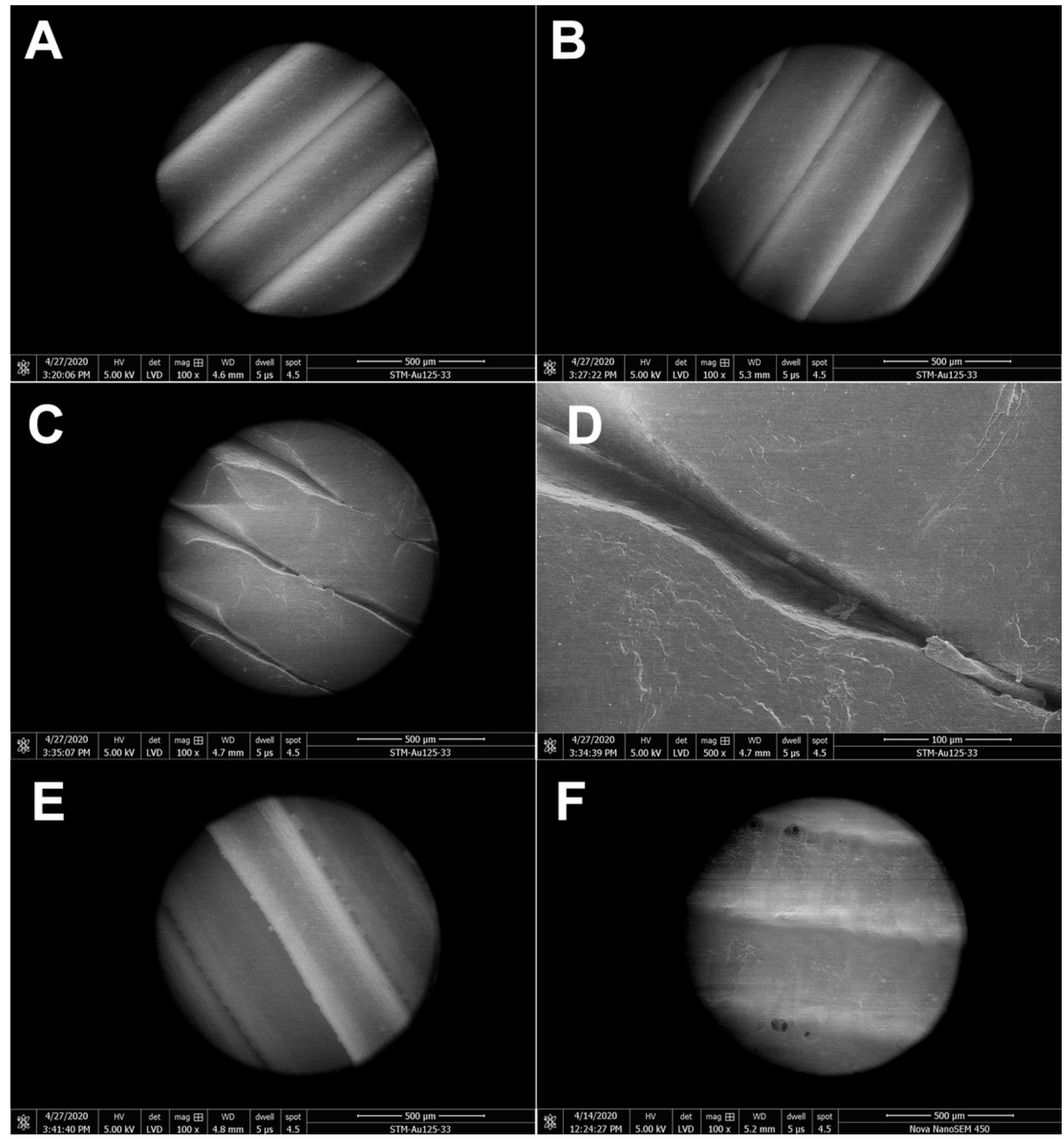




\section{Table $\mathbf{1}$ (on next page)}

PLA material contaminated by Staphylococcus epidermidis, Escherichia coli and Candida albicans, untreated or treated with ethanol, isopropanol or sodium hypochlorite.

Results are expressed in $\mathrm{CFU} / \mathrm{mL}$, as the mean of triplicate tests. Untreated samples indicate the $\mathrm{CFU} / \mathrm{mL}$ count present on contaminated carriers. 


\begin{tabular}{lrrrr}
\multicolumn{4}{c}{ Colony forming units on PLA material after contamination by bacteria and yeast (CFU/mL) } \\
\cline { 2 - 5 } & untreated & ethanol & isopropanol & $\begin{array}{r}\text { sodium } \\
\text { hypochlorite }\end{array}$ \\
S. epidermidis & $1 \times 10^{5}$ & 0 & 1 & 0 \\
E. coli & $1 \times 10^{5}$ & 0 & 1 & 0 \\
C. albicans & $4 \times 10^{4}$ & 0 & 0 & 0
\end{tabular}

1 


\section{Table 2 (on next page)}

PLA material contaminated by SARS-CoV-2 and HAdV, untreated or treated with ethanol, isopropanol or sodium hypochlorite.

Results are expressed in $10^{3} \mathrm{IU} / \mathrm{mL}$, as the mean of triplicate tests. Untreated samples indicate the virus count on contaminated carriers in $\mathrm{IU} / \mathrm{mL}$. 


\begin{tabular}{lrrrr}
\hline \multicolumn{4}{c}{ Virus titers recovered from PLA material after contamination by virus $\left(10^{3} \mathrm{IU} / \mathrm{mL}\right)$} \\
\hline untreated & ethanol & isopropanol & $\begin{array}{r}\text { sodium } \\
\text { hypochlorite }\end{array}$ \\
SARS-CoV-2 & 114 & 0 & 0 & 0 \\
HAdV & 338 & 0.8 & 4.7 & 0 \\
\hline
\end{tabular}

1 


\section{Table 3(on next page)}

Natural contamination of PLA masks worn by test persons, and subsequently treated with ethanol (bacterial count expressed as CFU/mL). 


\begin{tabular}{lrrrr}
\hline \multicolumn{5}{c}{ Effect of ethanol on disinfection of PLA masks from natural microbial contamination } \\
& \multicolumn{4}{c}{ (CFU/mL) } \\
& $\begin{array}{r}\text { inner surface } \\
\text { (untreated) }\end{array}$ & $\begin{array}{r}\text { inner surface } \\
\text { (after } \\
\text { disinfection) }\end{array}$ & $\begin{array}{r}\text { outer surface } \\
\text { (untreated) }\end{array}$ & $\begin{array}{r}\text { outer surface } \\
\text { (after } \\
\text { disinfection) }\end{array}$ \\
\cline { 2 - 5 } PLA mask 1 & 7000 & 19 & 85 & 0 \\
PLA mask 2 & 257 & 0 & 153 & 0 \\
PLA mask 3 & 108 & 2 & 59 & 2 \\
average & 2455 & 7 & 99 & 1 \\
\hline
\end{tabular}

